\title{
La escritura, lenguaje vivo en el aula: una experiencia de autoformación docente en didáctica
}

\author{
Sonia Álvarez García' \\ sonialalvarezgarcia@gmail.com \\ María del Pilar Cruz Arias $^{3}$ \\ mapicruari@gmail.com
}

GRUPO SEMILLANDO

Adriana Lucia Sánchez García²

adribastidas@hotmail.com

Nubia Ginett Herrera Roa ${ }^{4}$

Ilwvia@yahoo.com

Consuelo Bellón Morales ${ }^{5}$

cobemo19@hotmail.com

\section{RESUMEN}

El proyecto surge de una mirada crítica de las prácticas y concepciones sobre los procesos de enseñanza y aprendizaje de la escritura en el aula de primer ciclo. Esta reflexión condujo a iniciar un proceso de investigación que permitiera articular las concepciones teóricas y las realizaciones del aula. Uno de los primeros resultados de este proceso es la transformación de la reflexión sobre las prácticas en acciones investigativas entre las cuales se destacan las siguientes: la lectura de la realidad, la formulación del proyecto de investigación como orientador de la práctica pedagógica, el abordaje de la investigación en el aula y la formulación de una propuesta didáctica para el desarrollo de la escritura significativa en el primer ciclo.

\section{Palabras Clave}

Proyecto pedagógico, escritura, significación, proyectos de aula, didáctica.

\section{ABSTRACT}

The project Teaching Writing, Language Alive in the Classroom: An Experience of Teacher Self-Training in Didactic, developed by the sEMILLANDO group, comes from a critical look of our practices and concepts about writing and its teaching and learning processes in the first cycle of education. This reflection led us to initiate an investigation in our schools which would allow us to articulate the theoretical conceptions and achievements in the classroom. One of the first results of this process is the transformation of the reflection on the practices in investigative actions, among which these stand out: reading reality, formulating the research project as a guide of the pedagogical practices, the approach to research in the classroom and the formulation of a didactic proposal for the development of writing in the first season.

\section{KEYWORDS}

Educational project, significant writing, classroom projects, didactic.

\footnotetext{
IED Salitre.

IED Salitre.

Colegio La Paz ced.

4 IED Rufino José Cuervo.

5 IED Alfredo Iriarte.
} 


\section{Introducción}

$\mathrm{E}$ 1 presente artículo aborda unà experiencia de investigación que indaga por las huellas dejadas por nuestras concepciones y prácticas de la enseñanza de la escritura en los grados iniciales. Se han replanteado las prácticas pedagógicas de aula para iniciar la búsqueda de respuestas a la pregunta de cómo abordar la enseñanza y el aprendizaje de la escritura, en estos grados, como proceso de construcción de la significación. En este artículo se describen las acciones investigativas que se han adelantado para dar respuesta al interrogante investigativo.

En la primera acción de investigación se presenta.la lectura de la realidad como un proceso de caracterización de las concepciones desde los aspectos teóricos como desde las prácticas en el aula con respecto a la escritura. Esto permitió entender el papel del docente en los procesos de desarrollo de la significación como eje fundamental para el aprendizaje de la escritura de los niños en los grados iniciales.

La segunda àcción consistió en la formulación de un proyecto de investigación que posibilitó orientar la práctica pedagógica. La mirada comprensiva de la praxis pedagógica del grupo permitió establecer como problema de investigación la indagación sobre las estrategias didácticas para la enseñanza y el aprendizaje significativo del lenguaje escrito en los niños de grados iniciales a través de la pedagogía de proyectos. A partir de este interrogante, se formularon los objetivos de la investigación, se definieron los referentes teóricos y metodológicos, las acciones investigativas y los instrumentos y procedimientos para el desarrollo de la propuesta.

En la tercera acción se presenta la propuesta de abordaje de la investigación en el aula de preescolar. Se proponen los proyectos de aula como una alternativa pedagógica en ciclo inicial que permite hacer de la escritura ùna actividad de significación y un espacio de implementación de la propuesta de investigación en didáctica de la escritura.

Por último, a partir de la experiencia investigativa se expone la propuesta didáctica para el desarrollo de la escritura en el primer ciclo fundamentada en una concepción de didáctica como proceso de investigación de las concepciones y prácticas en el aula.

\section{La lectura de la realidad como práctica reflexiva}

Esta acción investigativa estuvo centrada en la caracterización de las concepciones sobre escritura, expresadas en las prácticas en los entornos escolares, incluyendo las aulas de las integrantes del grupo. Estas prácticas se caracterizaban por considerar a los niños como sujetos activos en el aprendizaje de la escritura. En efecto, los niños, por encontrarse en una cultura de lo impreso, construyen hipótesis acerca de qué y cómo se escribe. Desde nuestras prácticas esta concepción se reflejaba entonces en la adquisición del símbolo a partir de la propuesta de $\mathrm{Fe}$ rreiro (1988) en lo concerniente a las hipótesis de escritura, a saber: de cantidad, variabilidad, silábica y fonética. Una vez el niño adquiría el símbolo, se pasaba a las actividades de dictado y se establecía el copiado como sinónimo de escribir.

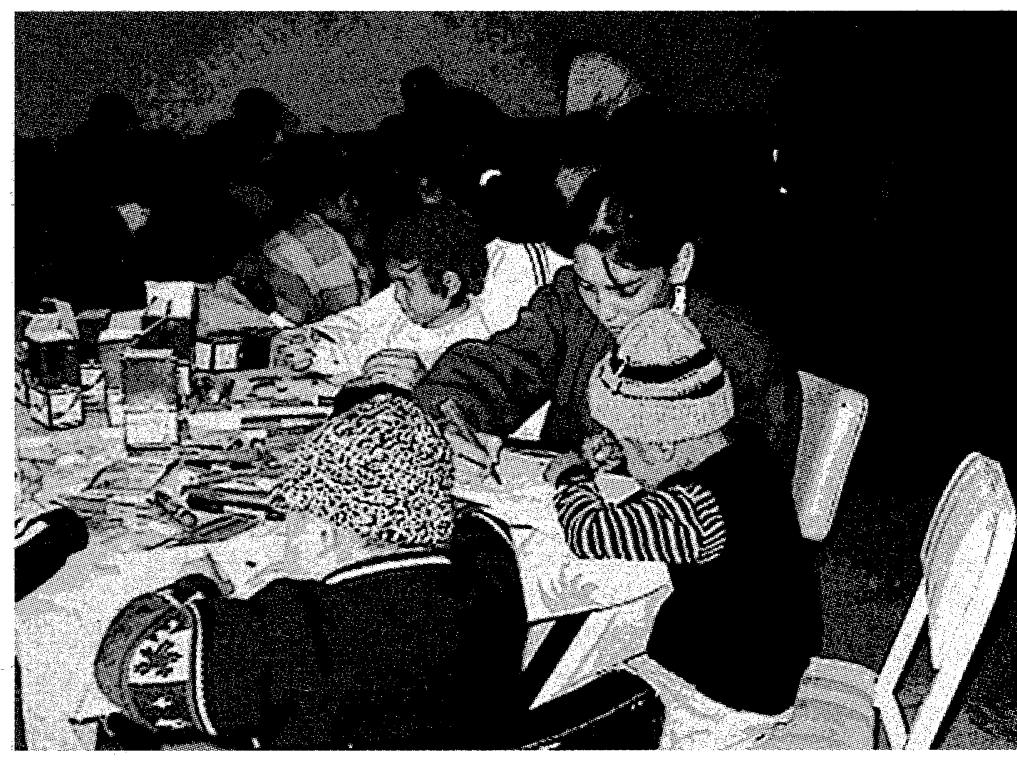

La reflexión sobre estos procedimientos permitió comprender que esta manera de asumir el proceso de enseñanza y aprendizaje de la escritura desvinculaba las representaciones propias de los niños de las representaciones formales de escritura impuestas en el aula, si se tienen en cuenta las siguientes consideraciones: 
- La concepción de escritura separaba la oralidad, el gesto, el juego y el dibujo de la escritura. Es decir, no se alcanzaba a ver que estas actividades le sirven al niño para representar sus ideas y pensamientos, son sus primeras "escrituras" que más tarde ayudan al niño a tomar conciencia del carácter de representación de esta forma del lenguaje.

- Los proyectos de aula no se vinculaban con la tarea de escribir para desarrollar la comunicación, el pensamiento y la creación como aspectos fundamentales del desarrollo del niño. Las ocasiones donde se brindaba espacio para escribir eran espontáneas, sin ningún tipo de intervención por parte de las docentes para apoyar el proceso de escritura de los textos ya que se tenían falencias sobre los conocimientos lingüísticos de los diversos tipos de textos y sobre la didáctica específica de la escritura.

- Por otra parte, las dinámicas de interacción en el aula muestran el predominio de la acción de las docentes, los diálogos y cuestionamientos no generaban desequilibrio y transformación de los conocimientos acerca de la escritura. La interacción entre niños, a través del trabajo en grupo para escribir, no existía, por lo tanto, no se propiciaba el aprendizaje cooperativo y la potenciación de la zona de desarrollo próximo. En términos generales, no se propiciaban situaciones de verificación, de compartir o buscar soluciones a problemas cognitivos generados a partir de la comprensión y producción de textos.

- En cuanto a la sistematización de la experiencia, ésta se realizaba a través del diario de campo. Sin embargo, en este documento sólo se registraban las actividades planeadas de lectura y escritura, sin articulación con los logros e indicadores que se querían alcanzar con la actividad, al nivel de saberes y/o competencias. Así mismo, los logros planteados para los grados iniciales, en escritura, sólo tenían en cuenta el gusto por la actividad, las etapas de adquisición del símbolo, la escritura de los nombres de los niños y el manejo del renglón, entre otros, sin referencia a los elementos que requiere llevar al niño a ponerse en contacto con la cultura de lo escrito.

- De igual forma, se consideraba la escritura como una actividad en la que no eran necesarios los conocimientos del contexto sociocultural para promoverla. Se desconocía que la escritura, como una actividad sociocultural compleja, requiere poner en juego varias competencias y hay que concienciar sobre ellas para lograr decidir qué se va a escribir y cómo se va a escribir.

Las anteriores constataciones sọbre la práctica de la escritura en el aula pusieron de manifiesto la brecha entre la teoría y la práctica y se constituyeron en el fundamento de las acciones investigativas, abordadas a continuación.

Se han replanteado
las prácticas pedagógicas
de aula para iniciar
la búsqueda de respuestas
a la pregunta de cómo
abordar la enseñanza
y el aprendizaje de la escritura,
en estos grados,
como proceso de construcción
de la significación.

\section{La formulación del proyecto de investigación como orientador de la práctica pedagógica}

La lectura de la realidad de la escritura en el aula permitió definir como interrogante investigativo general, el siguiente: ¿Cuáles son las estrategias didácticas para la enseñanza y el aprendizaje significativo del lenguaje escrito, en el ciclo inicial, a través de la pedagogía de proyectos?

En articulación con el anterior interrogante se propuso como objetivo general construir una ruta didáctica para la enseñanza y el aprendizaje significativo del lenguaje escrito, en el ciclo inicial, a través de la pedagogía de proyectos. 
Como objetivos específicos se definieron los siguientes:

- Promover espacios colectivos de autoformación docente para la construcción de concepciones teóricas y prácticas coherentes con las realidades de aula.

- Crear ambientes de enseñanza y aprendizaje holísticos en el aula, que favorezcan el desarrollo de la lengua escrita.

- Crear y adaptar instrumentos de evaluación del impacto de la investigación en escritura en los niños.

Para emprender las acciones investigativas y dar solución al problema encontrado se asumió la tarea de construir, en colectivo, referentes teóricos que permitieran investigar qué implicaba aprender la escritura desde la propuesta de la significacion en el aula. Así, se definieron como referentes conceptuales:

La comprensión de la escritura como una actividad específica del lenguaje que tiene por objeto la producción de significados a través de los textos escritos que va más allá de la codificación, permitiendo a los sujetos escritores la reelaboracion del pensamiento en la interaccion con otros ausentes o presentes, para recrear contextos culturalmente situados.

En segundo lugar, el planteamiento del aprendizaje desde una concepción interaccionista, lo cual significa concebirlo como un proceso por medio del cual hay una apropiación y negociación de significados que se da a través de distintos mecanismos de intersubjetividad (Sánchez, 2000). Estos significados los construye el ser humano al habitar una cultura, compartir su lenguaje y vivir con los otros. En conjunto, estudiantes, maestros y comunidad en general aportan su potencial de saberes, vivencias, emociones, intereses, inquietudes y necesidades a este proceso de intersubjetividad a través de prácticas comunicativas y a la transformación de los objetos de aprendizaje, comprometiéndose en un proceso continuo y sistemático de investigación (Jaimes y Rodríguez, 1996).

En tercer lugar, la asunción de la didáctica de la escritura como campo de la investigación sobre los procesos de enseñanza y aprendizaje del len- guaje escrito de manera significativa, procurando al máximo conservar la relación entre la escritura como objeto de enseñanza y como práctica social, para que luego los niños la usen realmente en diversos contextos extraescolares (Lerner, 2001).

En cuarto lugar, la experiencia investigativa sustentada en la pedagogía de proyectos como una alternativa de construcción de conocimiento tanto para el docente como para el estudiante. Para el docente porque una vez él analiza el contexto del aula y detecta las carencias, se plantea un problema de investigación a resolver y se lleva a cabo una propuesta de transformación pedagógica de aula y/o institucional. Para los estudiantes, por ser una pedagogía interaccionista que les permite articular la vida y la escuela en proyectos específicos que dan respuesta no sólo a determinados problemas del conocimiento, sino a las necesidades que surgen en la vida escolar y donde el lenguaje se constituye en un sistema de mediación cultural entre los maestros, los estudiantes y el conocimiento (Lozano, 1999).

En quinto lugar, la postulación de los proyectos de aula como una alternativa de construcción de conocimiento de docentes y estudiantes en el aula. Éstos surgen a partir de las inquietudes, intereses y necesidades tanto de los niños, maestros y de la institución misma en su contexto. En ellos se hace uso de la escritura como una actividad significativa para los niños, una actividad importante en sus vidas. Los proyectos de aula permiten que la actividad escritora se plantee desde el comienzo como una forma de lenguaje auténtica y compleja. A su vez, permiten a los niños concebirse a sí mismos desde los grados iniciales como escritores de textos con la competencia comunicativa necesaria para interactuar con otros mediante la escritura. El hecho de producir diversos tipos de textos, acordes con sus necesidades comunicativas, los obliga a desarrollar conciencia de la diversidad textual y la manera como circulan los textos en la sociedad, a reconocer la existencia de distintos propósitos en la comunicación escrita y a apropiarse de las formas específicas que asumen las tipologías textuales, además, les permite ubicarse como autores de textos dirigidos a lectores potenciales o reales (Lozano, 1999). 
A la par con los anteriores referentes teóricos, en especial la pedagogía de proyectos como una alternativa de construcción de conocimiento disciplinar, pedagógico y didáctico que pretende transformar las prácticas escritoras en el aula, se hizo necesario definir unos criterios metodológicos de investigación que permitieran observar y contrastar la pertinencia de la teoría en los contextos escólares.

En consecuencia, se retomó la teoría de la investigación-acción participativa (IAP) como una metodología coherente con el deseo de transformación de las prácticas en el aula. En nuestro caso, utilizamos elementos etnográficos como encuestas, observaciones y análisis de documentos para comprender los procesos de enseñanza y aprendizaje en la escritura. De allí nos planteamos una situación problemática que permite establecer una relación íntima entre la teoría de la escritura y la práctica en el aula, que arrojó como resultado la' construcción de una propuesta de transformación de las prácticas pedagógicas para mejorar dichos procesos en contextos específicos.

En términos generales la investigación de las prácticas ha generado un proceso de extrañamiento y admiración, por decir de algún modo, de las actuaciones de los niños y de las nuestras como docentes de aula, que permite readecuar las teorías y plantear nuevas formas de pensar y actuar en ellas, enriqueciendo la experiencia pedagógica propia y de la comunidad académica.

\section{El abordaje de la investigación en el aula}

Una vez determinado el problema de investigación se empezó a construir una propuesta de escritura significativa inmersa en los proyectos de aula y a observar sistemáticamente la relación entre las maneras de enseñar la escritura y las producciones escritas de los niños. Los proyectos de aula permitieron crear un espacio de investigación en donde se logró integrar el saber y la práctica pedagógica. En ellos el actuar pedagógico y didáctico del maestro se convirtió en objeto de investigación y, por lo tanto, la cohe- rencia entre la teoría y la práctica docentes fueron objeto de autoexploración al mismo tiempo.

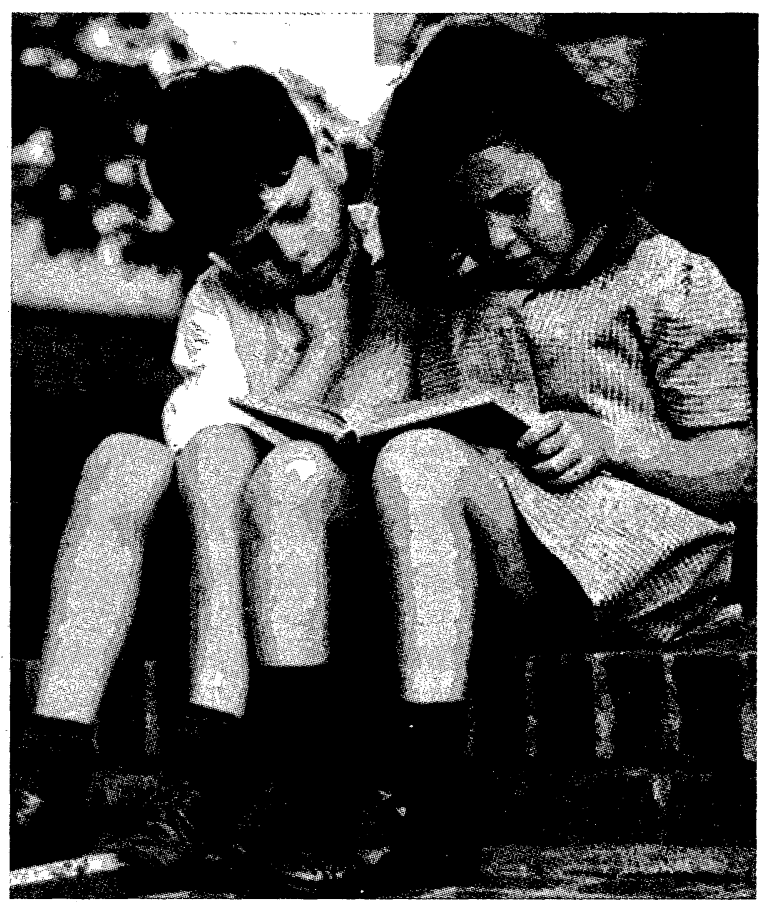

Los proyectos de aula permitieron conciliar los medios y los fines del proyecto de investigación. En la planificación y desarrollo se procuró generar situaciones de escritura contextualizadas que se desprendieran naturalmente de las necesidades de los los proyectos. Por ejemplo, ante la necesidad de visitar un museo existe la exigencia cultural de solicitar un permiso mediante una carta. A partir de estas necesidades, se abrió el espacio para pensar e implementar las estrategias didácticas que permitan a los niños hacer de la escritura una actividad de producción de significado. Al mismo tiempo, se posibilita la reflexión de las docentes acerca de la intervención pedagógica y didáctica con base en lo que hacen o dejan de hacer los niños cuando se realiza la actividad, propiciando el replanteamiento de las acciones.

En este proceso cobra importancia la sistematización de los proyectos de aula porque permite observar detenidamente la actividad de los niños, al escribir en situaciones reales de uso, y puntualizar la mirada investigativa sobre los distintos interrogantes formulados, para dar respuesta a la pregunta de investigación. Una vez recogidas, las diversas experiencias de aula son 
comparadas en el colectivo y se reflexiona sobre los procesos desarrollados y los resultados obtenidos. Esta acción investigativa ayuda a cuestionar el actuar, a ver las fortalezas y debilidades y, lo más importante, a problematizar y categorizar en colectivo las estrategias de enseñanza y aprendizaje del lenguaje escrito en el aula.

De la anterior problematización han surgido otros interrogantes investigativos que contribuyen a responder la gran pregunta y dan cuenta del avance del grupo en la identificación de los elementos constitutivos del problema de investigación. Éstos son:

- ¿Cómo se contribuye desde la oralidad a la producción de textos escritos?

- ¿Cómo y por qué favorecer la prehistoria del lenguaje escrito en la enseñanza de la escritura a los niños de grados iniciales?

- ¿Cómo y qué evaluar en los textos que producen los niños del ciclo inicial?

- ¿Cuáles son las estrategias de lectura que contribuyen a la producción escrita?

- ¿Cuál es la intención de la pregunta del maestro cuando la utiliza como apoyo para la producción escrita del niño en el ciclo inicial?

Abordar cada uno de los anteriores interrogantes condujo a la formulación de una propuesta para el desarrollo de la escritura en el primer ciclo.

\section{Formulación de una propuesta didáctica para el desarrollo de la escritura en el ciclo inicial}

Las anteriores reflexiones y constataciones a partir de la experiencia pedagógica e investigativa han permitido generar estrategias didácticas para la enseñanza y aprendizaje de la escritura. Se entienden las estrategias como acciones intencionadas del docente de aula que orientan y promueven el proceso de enseñanza y aprendizaje de la escritura como actividad de significación. Las estrategias que se proponen son el fruto de un proceso de investigación de las prácticas de aula, coherentes con planteamientos teóricos que plantean la necesidad de la interacción entre es- tudiantes, docentes y el objeto de estudio, es decir, la escritura.

Se parte del principio de que los niños ya tienen representaciones naturales de los procesos escritores, construidos mucho antes de la escolarización. En consecuencia, las estrategias propuestas tienen en cuenta la relación de complementariedad entre las representaciones primarias como el gesto, el juego y el dibujo y las actividades del lenguaje de la oralidad, esto es, hablar y escuchar, y las de escritura, es decir, leer y escribir. Igualmente se reconoce la importancia de lograr la autonomía de los niños en la producción de textos completos y diversos. A continuación se abordan algunas de las estrategias propuestas, derivadas de la investigación en el aula:

\section{Estrategia 1. Investigar como estrategia para aprender de la escritura de los niños}

Con esta experiencia se aprende que enseñar a escribir en el aula de ciclo inicial, más que la aplicación de un procedimiento, es un proceso de reflexión continuo sobre los logros o dificultades de los niños en escritura. El docente como practicante reflexivo se constituye en mediador en el proceso de construcción de la escritura como sistema de representación y significación. Esta reflexión, al ser crítica, sistematizada y sustentada, no sólo desde la experiencia de aula sino a partir de la teoría, se puede constituir en un proceso de investigación.

\section{Estrategia 2. Jugar y representar}

La propuesta reconoce el juego, el gesto y el dibujo como un sistema primario de representaciones. El niño llega a la escuela con este sistema, el cual es la base para futuros procesos de representación como la escritura. Definimos como sistema primario las primeras herramientas utilizadas para poder comunicar una intención. Vigotsky las define como procesos psicológicos de primer orden, ya que al constituirse como representaciones guardan una relación estrecha con lo que representan: Estas actividades le permiten al niño liberarse de su percepción para pasar al plano de la imaginación y convertirlas en 
actividades intelectuales conscientes que lo llevan a actuar por el significado de las situaciones vividas (2000).

\section{Estrategia 3. Leer para escribir. La interrogación de textos}

Se propone la interrogación de textos como una estrategia anterior a la escritura de un texto, con la intención de observar cómo se escriben, en el contexto cultural, los textos que necesitamos para lograr nuestros objetivos en el marco de un proyecto. $\mathrm{El}$ propósito de la interrogación de textos es la búsqueda de pistas para comprender la totalidad del texto y usar luego esas mismas pistas para escribir el texto que se necesita. Mediante esta interrogación, la maestra se propone que el niño identifique la función de los textos insertos en la cultura, los tipos de textos, los parámetros de la situación de comunicación, las siluetas de los textos y los tipos de discursos (Jolibert, 1998).

Esta estrategia requiere una buena preparación por parte del maestro, porque implica que sean los niños los que interroguen el texto y no el maestro quien interrogue a los niños sobre el texto. Esto significa que son los niños quienes deben asumir el control de la lectura, los que construyen las hipótesis, confrontan el texto y construyen su significado.

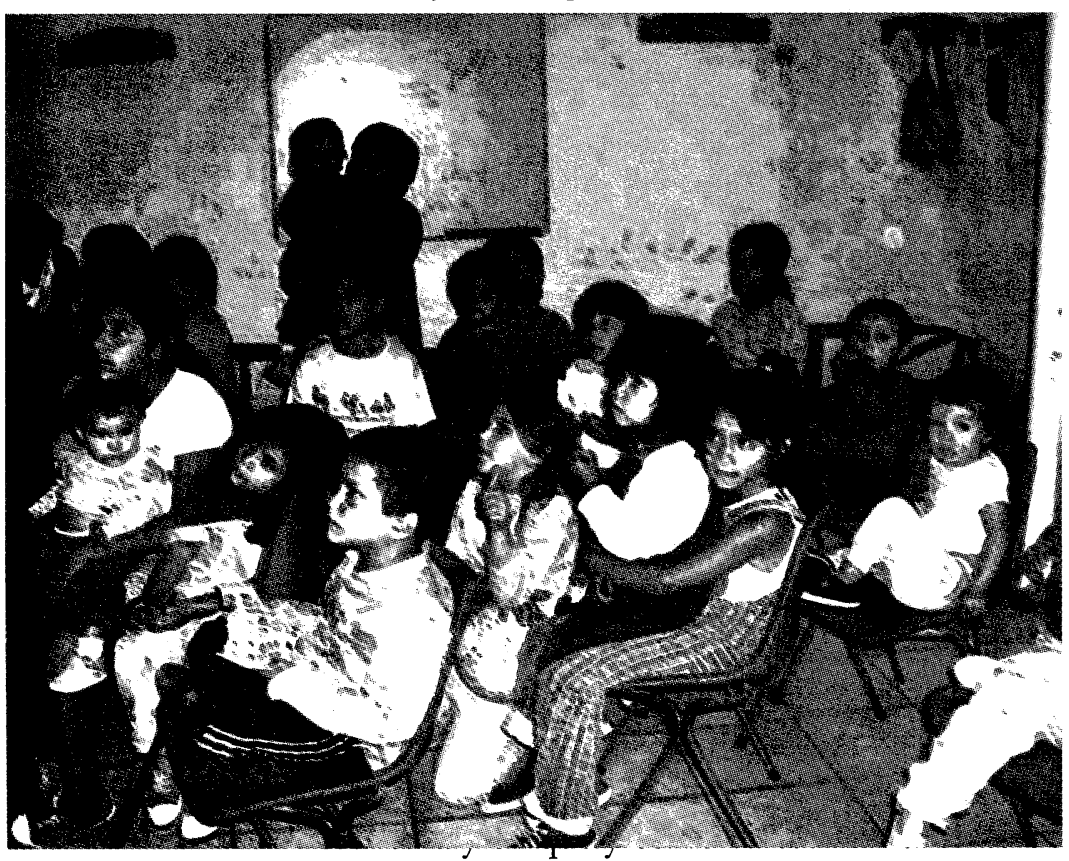

acuerdo con su intencionalidad y los usos de los textos que circulan en su comunidad.

\section{Estrategia 5. Acompañar al niño en el momento crítico de la toma del lápiz y la escritura autónoma}

La escritura involucra elementos materiales para cuyo manejo el niño requiere dominios específicos. En el momento en que el niño toma el lápiz para escribir el texto de su propia mano se hace necesario el acompañamiento, pues el acto de escritura exige varias competencias al mismo tiempo, que tienen que ver con el uso de instrumentos y la construcción de la significación.

La experiencia demuestra que es necesario el apoyo para que el niño logre producir un texto con significado, es decir, que pueda comunicar lo que quiere decir por escrito partiendo desde donde él quiere. Este acompañamiento realizado mediante preguntas conlleva a que el niño tome conciencia sobre los diferentes aspectos que comprometen la escritura de un texto. En este momento la oralidad acompaña el proceso de escritura. Cuando el niño empieza su escrito, escribe como habla. Hemos visto que lo primero que comunica son las intenciones, sin pensar en cómo y en qué momento las escribe; como habla con ideas completas escribe ideas completas.

\section{Estrategia 6. Evaluar los textos escritos y ganar en autonomía}

La observación y reflexión sobre el trabajo del aula y la investigación demostró que la evaluación de los textos es una estrategia que ayuda a la producción textual. Se concibe la evaluación como un proceso continuo y sistemático de observación y análisis de los textos escritos, que permite a los niños formarse como productores de textos reales al desarrollar la conciencia acerca de su proceso escritor. Esta conciencia lleva a los niños a reflexionar sobre qué se escribe y cómo se escribe.

Para el proceso de evaluación de la producción de un texto completo se construyó, junto con los niños, una rejilla de evaluación que les permite producir y evaluar un texto autónomamente de acuerdo con los criterios definidos. Con estas rejillas se evalúan diversos tipos de textos tales como cartas, recetas, afiches, carteles, cuen- 
tos, poemas, libros y canciones, entre otros. $\mathrm{La}$ evaluación textual permite que el niño sienta el impacto de la producción de su texto y sea consciente de las consecuencias de la escritura.

\section{Estrategia 7. La adquisición del código:} el punto de llegada de la escritura con sentido

\section{En el caso del ciclo inicial, el niño empieza a interesarse por el código alfabético y por otros aspectos que hacen parte, también, de la competencia textual: los signos de puntuación y las tildes, entre otros; en suma: la ortografía.}

El posibilitar la escritura como lenguaje vivo en el aula conduce a los niños a darse cuenta de que existe un sistema alfabético que le posibilita ser comprendida por otros para que su texto cumpla los efectos esperados. En el caso del ciclo inicial, el niño empieza a interesarse por el código alfabético y por otros aspectos que hacen parte, también, de la competencia textual: los signos de puntuación y las tildes, entre otros; en suma: la ortografía. En este momento es función del docente ofrecer los andamiajes necesarios para que los niños construyan el código de escritura. Puede ser mediante preguntas acerca de cómo se escriben las palabras en el marco de la escritura de un texto o por actividades estructuradas que resuelvan las inquietudes que tengan los mismos niños acerca de la apropiación del sistema. Ó

\section{Bibliografía}

Ferreiro, E. (1988), Los sistemas de escritura en el desarrollo del niño, Bogotá, Siglo XXI.

Jaimes, G. y Rodríguez, M. E. (1996), Lenguaje y mundos posibles, Bogotá, Universidad Distrital-Colciencias.

Jolibert, J. (1998), Interrogar y producir textos auténticos: Vivencias en el aula, Santiago de Chile, Dolmen.

Lozano Rodríguez, I. (1999), Proyecto pedagógico, proyectos de aula y procesos de apropiación de la lectura y la escritura, memorias del Congreso Colombiano de Lectoesctitura en Lengua Materna y Lengua Extranjera para un Nuevo Siglo, Bogotá, Universidad Distrital Francisco José de Caldas.

Lerner, D. (2001), Leer y escribir en la escuela: lo real, lo posible y lo necesario, Fondo de Cultura Económica, México.

Sánchez, Y. (2000), Vygotsky boy, Bogotá, Unibiblos.

Vygotsky, Lev. (2000), El desarrollo de los procesos psicológicos superiores, Barcelona, Crítica. 